\title{
Putting the pressure on endocytosis in the kidney
}

\author{
Dorien Van Giel and Rudi Vennekens
}

Laboratory of Ion Channel Research, TRP Research Platform Leuven, VIB center for Brain and Disease Research, Department of Cellular and Molecular Medicine, Faculty of Medicine, KU Leuven, Belgium.

\section{In Science Signaling, Gualdani and colleagues provide evidence that the ion channel TRPV4 functions as a mechanosensor in the renal proximal tubules and show that TRPV4 activity modulates protein reabsorption.}

In the kidneys, blood is filtered through the glomeruli. About $70 \%$ of the water, solutes and virtually all of the proteins such as albumin that pass through the glomerular filtration barrier are reabsorbed by the proximal tubule (PT) epithelial cells. The urinary albumin level is thus reflected by the combined contribution of the filtration and reabsorption activity of the glomeruli and PT, respectively. Dysfunction of both processes may result in elevated excretion of albumin, causing proteinuria as seen in renal diseases of various etiologies. Moreover, an excess of albumin in the tubular lumen can trigger epithelial cells to function as inflammatory and fibrogenic cells, accelerating the progression of renal disease. The reduction of albuminuria is therefore a target as such to prevent deterioration of renal function. ${ }^{1}$

The PT is subject to mechanical forces, induced by the entry of the ultrafiltrate in the tubular lumen. This includes two types of forces, being shear stress on the apical membrane generated by fluid flow and radial stretch caused by a raise in intraluminal pressure. Studies have indicated that PT function is modulated by the amount of ultrafiltrate and therefore suggest the presence of mechanosensory mechanism. ${ }^{2}$ One proposed action was the enhancement of endocytosis in PT cells induced by fluid flow in a $\mathrm{Ca}^{2+}$ - and cilium-dependent mechanism. ${ }^{3}$ However, recent findings refute that mechanosensation in the cilium operates via $\mathrm{Ca}^{2+}$ signaling. ${ }^{4}$ Alternatively, radial stretch could be sensed by the cytoskeleton, adhesion proteins or stretch-sensitive ion channels and subsequently modify the endocytotic function of PT cells, but the mechanism through which this occurs remained largely unexplored.

In Science Signaling, Gualdani et al. ${ }^{5}$ provide evidence that mechanical deformation of PT epithelial cells modulates their endocytotic function, and that the $\mathrm{Ca}^{2+}$ permeable ion channel TRPV4 operates as a sensor for radial stretch in the underlying mechanotransduction pathway. 
In their study, Gualdani et al. identified the functional presence of TRPV4 in the basolateral membrane of murine primary PT cells. The authors found that the channel can be activated by the specific agonist GSK1016790A, hypotonic solution and by mechanical stretch generated through silicone stretchable chambers. In contrast, the application of flow-induced shear stress did not evoke TRPV4-dependent $\mathrm{Ca}^{2+}$ responses. Furthermore, cells lacking TRPV4 showed a decrease in the uptake of FITC-labeled albumin and dextran upon stimulation with GSK1016790A or mechanical stretch. Thus, these data together indicate that TRPV4 plays a critical role in the transduction of mechanical stimuli leading to a change in albumin endocytosis.

Gualdani et al. further underlined the relevance of their findings by investigating whether the presence of TRPV4 affects the observed degree of proteinuria in mice challenged with experimental conditions that mimic nephropathies and increased permeability of the glomerular filter. Notably, they found that mice lacking TRPV4 globally or specifically in the PT exacerbated proteinuria whereas urinary levels of glucose and phosphate, also reabsorbed by the PT, remained unaffected.

The main contribution of the study by Gualdani et al. is the identification of a specific molecular sensor responsible for the conversion of a mechanical stimulus into functional alterations of PT cells. The observations also explain a mechanism that links mechanosensation and intracellular $\mathrm{Ca}^{2+}$ signals without involvement of the primary cilium. These data subsequently open new perspectives for therapy in proteinuria, as the possibility to interfere with an upstream event that drives albuminuriainduced decline of renal function is expected to have greater efficacy than targeting downstream manifestations.

An important question that remains to be answered is whether TRPV4-mediated albumin endocytosis plays a role in proteinuria observed in human kidney diseases. Validating the translatability of these study results to human cell models and/or patients will be crucial to evaluate the relevance for possible treatment strategies. Thus far, TRPV4 was, in contrast to this study, described to modulate flow-induced $\mathrm{Ca}^{2+}$ responses in renal epithelial cells. ${ }^{6}$ However, this was found in human cell models of the distal nephron and similar studies in PT epithelial cells are lacking. Further, mutations in TRPV4 have been associated with Charcot-Marie-Tooth disease (CMT), but also skeletal dysplasias. ${ }^{7}$ Interestingly, in CMT patients, an increased prevalence of glomerulopathies has been documented, which is associated with proteinuria. ${ }^{8}$ It could therefore also be of interest to investigate a possible involvement of TRPV4-modulated protein reabsorption in these pathologies. 
1. Birn, H. and E.I. Christensen, Renal albumin absorption in physiology and pathology. Kidney Int, 2006. 69(3): p. 440-9.

2. Raghavan, V. and O.A. Weisz, Discerning the role of mechanosensors in regulating proximal tubule function. Am J Physiol Renal Physiol, 2016. 310(1): p. F1-5.

3. Raghavan, V. and O.A. Weisz, Flow stimulated endocytosis in the proximal tubule. Curr Opin Nephrol Hypertens, 2015. 24(4): p. 359-65.

4. Delling, M., et al., Primary cilia are not calcium-responsive mechanosensors. Nature, 2016. 531(7596): p. 656-60.

5. Gualdani, R., et al., Mechanical activation of TRPV4 channels controls albumin reabsorption by proximal tubule cells. Sci Signal, 2020. 13(653).

6. Mamenko, M., et al., Deciphering physiological role of the mechanosensitive TRPV4 channel in the distal nephron. Am J Physiol Renal Physiol, 2015. 308(4): p. F275-86.

7. Nilius, B. and T. Voets, The puzzle of TRPV4 channelopathies. EMBO Rep, 2013. 14(2): p. 15263.

8. De Rechter, S., et al., Charcot-Marie-Tooth: are you testing for proteinuria? Eur J Paediatr Neurol, 2015. 19(1): p. 1-5.

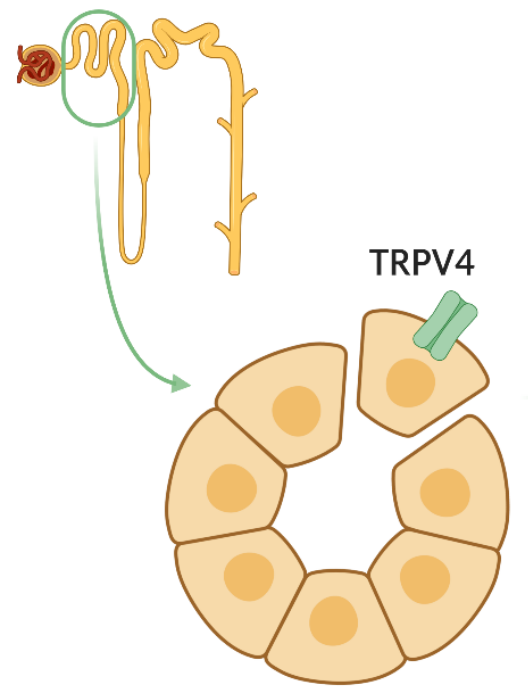

Cell stretching

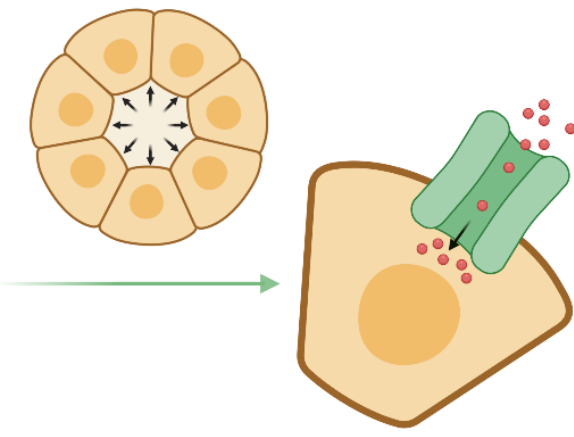

$\mathrm{Ca}^{2+}$ influx

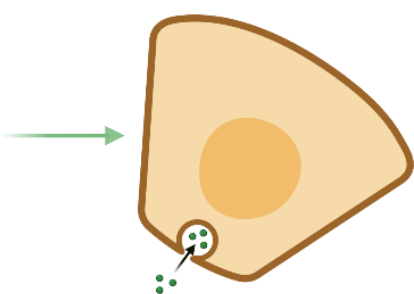

Endocytosis

Figure 1 | TRPV4 is a mechanosensor in the PT that controls protein endocytosis. Gualdani et al. show that $\mathrm{Ca}^{2+}$ responses induced by radial stretch of the PT are depending on TRPV4 activity and drive endocytosis of albumin. Created with BioRender.com. 